\title{
RENEWAL: NEW ASPECTS OF ACCELERATION AND TRANSPORT OF SOLAR ENERGETIC PARTICLES (SEPS) FROM THE SUN TO THE EARTH
}

Stephen W. Kahler

31 October 2014

Final Report

APPROVED FOR PUBLIC RELEASE; DISTRIBUTION IS UNLIMITED

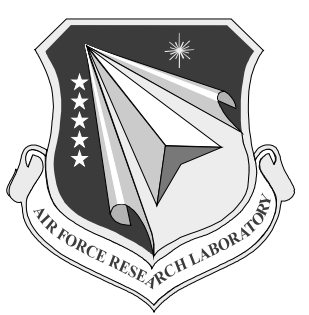

AIR FORCE RESEARCH LABORATORY Space Vehicles Directorate 3550 Aberdeen Ave SE KIRTLAND AIR FORCE BASE, NM 87117-5776 


\section{DTIC COPY}

\section{NOTICE AND SIGNATURE PAGE}

Using Government drawings, specifications, or other data included in this document for any purpose other than Government procurement does not in any way obligate the U.S. Government. The fact that the Government formulated or supplied the drawings, specifications, or other data does not license the holder or any other person or corporation; or convey any rights or permission to manufacture, use, or sell any patented invention that may relate to them.

This report was cleared for public release by the 377 ABW Public Affairs Office and is available to the general public, including foreign nationals. Copies may be obtained from the Defense Technical Information Center (DTIC) (http://www.dtic.mil).

AFRL-RV-PS-TR-2014-0213 HAS BEEN REVIEWED AND IS APPROVED FOR PUBLICATION IN ACCORDANCE WITH ASSIGNED DISTRIBUTION STATEMENT.

//SIGNED//

Dr. Stephen Kahler

Project Manager, AFRL/RVBXS
//SIGNED//

Glenn M. Vaughan, Colonel, USAF

Chief, Battlespace Environment Division

This report is published in the interest of scientific and technical information exchange, and its publication does not constitute the Government’s approval or disapproval of its ideas or findings. 


\section{REPORT DOCUMENTATION PAGE}

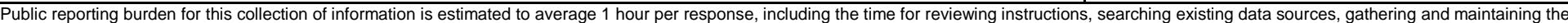

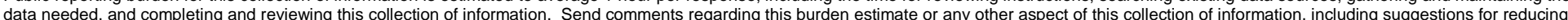

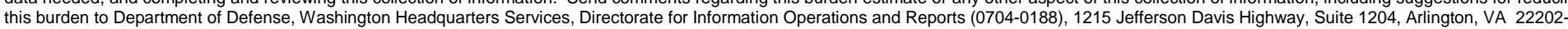

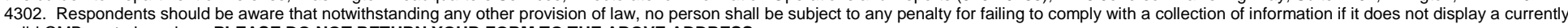
valid OMB control number. PLEASE DO NOT RETURN YOUR FORM TO THE ABOVE ADDRESS.

1. REPORT DATE $(D D-M M-Y Y Y Y) \quad$ 2. REPORT TYPE

30-10-2014

Final Report

4. TITLE AND SUBTITLE

Renewal: New Aspects of Acceleration and Transport of Solar Energetic Particles (SEPs)

from the Sun to the Earth

\section{DATES COVERED (From - To) \\ 1 Oct 2011 to 30 Sep 2014}

5a. CONTRACT NUMBER

\section{5b. GRANT NUMBER}

5c. PROGRAM ELEMENT NUMBER

$61102 \mathrm{~F}$

6. AUTHOR(S)

Stephen W. Kahler

\section{PERFORMING ORGANIZATION NAME(S) AND ADDRESS(ES)}

Air Force Research Laboratory

Space Vehicles Directorate

3550 Aberdeen Avenue SE

Kirtland AFB, NM 87117-5776

9. SPONSORING / MONITORING AGENCY NAME(S) AND ADDRESS(ES)

5d. PROJECT NUMBER

2301

5e. TASK NUMBER

PPM00018000

5f. WORK UNIT NUMBER

EF121233

8. PERFORMING ORGANIZATION REPORT

NUMBER

AFRL-RV-PS-TR-2014-0213

10. SPONSOR/MONITOR'S ACRONYM(S)

AFRL/RVBXS

11. SPONSOR/MONITOR'S REPORT NUMBER(S)

\section{DISTRIBUTION / AVAILABILITY STATEMENT}

Approved for Public Release; distribution is unlimited. (377ABW-2014-1031 dtd 13 Jan 2015)

\section{SUPPLEMENTARY NOTES}

\section{ABSTRACT}

This report summarizes the research results of this task which were published in eleven refereed journal articles. The seven basic topics are: (1) Effects of coronal holes on properties of SEP events (Kahler et al., 2013, 2014); (2) Size distributions and scaling laws of solar flares and SEP events (Cliver et al., 2012; Kahler, 2013); (3) SEP event timescales and associated CME properties (Kahler, 2013); (4) Comparison of the intensities and energies of gradual SEP events with the dynamical properties of associated CMEs (Kahler and Vourlidas, 2013); (5) The roles of interacting CMEs and solar wind streams in SEP events (Kahler and Vourlidas, 2014a,b); (6) The cosmogenic nuclide event of 775 AD (Cliver et al., 2014) ; and (7) Abundance enhancements and CME associations of Fe-rich impulsive SEP events (et al., 2014a,b) .

\section{SUBJECT TERMS}

Sun, Solar Flares, Coronal Mass Ejections, Particle Acceleration, Solar Energetic Particles

\section{SECURITY CLASSIFICATION OF:}

\section{a. REPORT}

Unclassified

\section{b. ABSTRACT}

Unclassified

\section{LIMITATION} OF ABSTRACT

c. THIS PAGE

Unclassified

\section{NUMBER} OF PAGES

Unlimited
22 19a. NAME OF RESPONSIBLE PERSON

Dr. Stephen W. Kahler

19b. TELEPHONE NUMBER 
This page is intentionally left blank.

Approved for public release; distribution is unlimited. 


\section{Table of Contents}

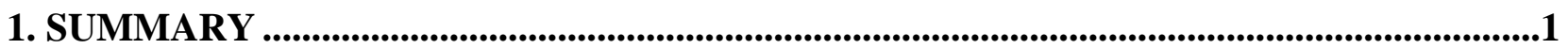

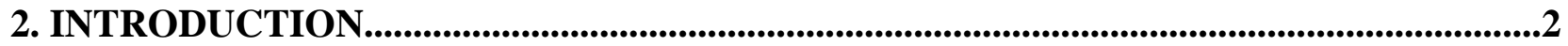

3. METHODS, ASSUMPTIONS, AND PROCEDURES .................................................................

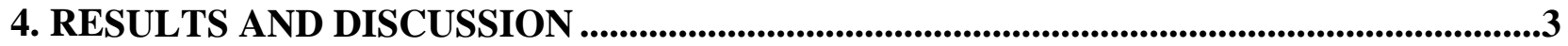

4.1. Effects of Coronal Holes on Properties of SEP Events .........................................................................3

4.2. Size Distributions and Scaling Laws of Solar Flares and SEP Events ............................................5

4.3. SEP Event Timescales and Associated CME Properties .....................................................................6

4.4. Comparison of the Intensities and Energies of Gradual SEP Events with the Dynamical Properties of Associated CMEs .............................................................................................................8

4.5. The Roles of Interacting CMEs and Solar Wind Streams in SEP Events ......................................9

4.6. The Cosmogenic Nuclide Event of 775 AD ....................................................................................... 11

4.7. Abundance Enhancements in Impulsive SEP Events ........................................................................... 11

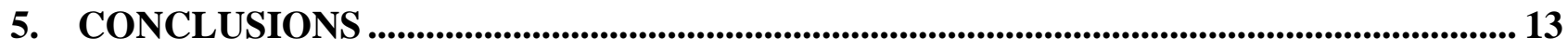

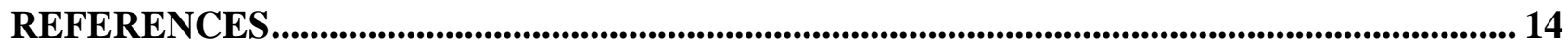




\section{List of Figures}

1. Schematic Diagram of CME Deflection by a Coronal Hole...............................................................4

2. SOHO EIT Image with Coronal Holes and FPA Vector .........................................................................4

3. The Coronal Mass Ejection Angles MPA Versus fPA............................................................................ 6

4. Plots of Size Distributions of Solar Flares and SEP Events ...........................................................6

5. Plot of SEP Onset and Rise Time Medians versus CME Speeds and Widths................................7

6. Plot of SEP Duration Time Medians versus CME Speeds and Widths ..........................................7

7. Plots of the Peak $20 \mathrm{MeV}$ Proton Intensities versus Three Sets of CME Speeds ........................9

8. Plots of SEP Event Log Energies versus Two Different CME Log Energies ................................9

9. Plots of $\log$ Ip versus $\log 2 \mathrm{MeV}$ proton backgrounds ....................................................................10

10. Plots of $\log$ Ip versus $\log$ CME speeds .....................................................................................10

11. Plots of the Power of A/Q versus X-ray Flare Peak and Fluence ................................................12 


\section{SUMMARY}

This report discusses work to analyze observations of solar energetic particle (SEP) events carried out under the aegis of a task funded by the Air Force Office of Scientific Research. The current task described here was a renewal of a completed three-year AFOSR task entitled "Understanding New Elements of Acceleration and Transport of Solar Energetic Particles (SEPs) from the Sun to the Earth". The goal is an improved understanding of the genesis and characteristics of SEP events to provide tools for future forecasts of SEP events. Under the task funding 11 papers were published in the refereed literature and are cited in the reference list.

The work carried out here is organized into seven sections, each focused on a particular theme dealing with the broad topic of SEP events and associated flares and coronal mass ejections (CMEs). The first research topic is the role of solar coronal holes (CHs) on the timing and intensities of 41 observed gradual $\mathrm{E} \sim 20 \mathrm{MeV}$ SEP events with CME source regions within $20^{\circ}$ of solar central meridian. We found no systematic $\mathrm{CH}$ effects on SEP event intensity profiles. We also found no correlation between the CME leading-edge measured position angles (MPAs) and SEP event properties, suggesting that the widths of CME-driven shock sources of the SEPs are much larger than the CMEs. Independently of the SEP event properties, we did, however, find evidence for significant $\mathrm{CME}$ deflections by $\mathrm{CH}$ fields in these events. We followed this work with a complementary statistical analysis of the same SEP events to look again for effects of CHs on SEP events, again finding unexpectedly that the presence of CHs does not appear to affect SEP intensities or event timescales.

The second theme was the size distributions of SEP event and associated solar flare intensities. A first study showed that the flatter size distribution SEP events relative to that of flare soft X-ray (SXR) events is primarily due to the fact that SEP flares are an energetic subset of all flares. The slopes ( $\alpha$ values) of power-law size distributions of the peak 1-8 Å fluxes of SXR flares associated with (a) $>10 \mathrm{MeV}$ SEP events (with peak fluxes $\geq 1 \mathrm{pr} \mathrm{cm}^{-2} \mathrm{~s}^{-1} \mathrm{sr}^{-1}$ ) and (b) fast CMEs were $\sim 1.3-1.4$ compared to $\sim 1.2$ for the peak proton fluxes of $>10 \mathrm{MeV}$ SEP events and $\sim 2$ for the peak 1-8 A fluxes of all SXR flares. A second study considered recent size distributions of SEP events compared with those of solar X-ray flares, which are distinctly steeper in slope. This has long been taken as evidence of a direct nonlinear physical connection between the processes producing the flares and those producing the SEP events. Four arguments were presented against the interpretation. In addition, the slope of the SEP event distribution was interpreted in terms of a recent model of fractal-diffusive self-organized criticality to provide a physical reason for the flatter SEP distributions.

The third theme was the relationship of CMEs to timescales of SEP events. We extended to all of solar cycle 23 a previous study to compare the timing characteristics of $217 \mathrm{E}=20 \mathrm{MeV}$ proton events with the parameters of their associated CMEs. The faster and wider CMEs resulted in longer rise and duration SEP timescales. The SEP event timescales were also organized as functions of solar source longitudes. The fourth theme was an attempt to compare total energies of some of the listed SEP events with those of associated CMEs. New methods to estimate the total SEP and CME energies were part of the analysis. In a comparison of 94 west limb CMEs and their SEP events we found that the SEP and CME energies are consistent with a linear scaling and that the SEP energies could be comparable to the CME energies. 
For the fifth theme we considered the sources of gradual SEP events. A popular model for enhanced SEP intensities involves several versions of interactions of the primary fast CMEs with prior CMEs occurring in the same coronal regions and within some hours earlier. We confirmed the statistical relationship but found an alternative possibility to understand the higher SEP intensities, namely that SEP events are larger when the solar activity, including CMEs, is increased. The population of a potential low-energy SEP seed population also increased along with the preceding CME occurrence. In a follow up to that study we looked at the characteristics of the solar wind streams in which the SEP events occurred. There was a definitely higher statistical intensity for the SEP events occurring in transient solar wind rather the fast or slow wind. A sixth topic was to determine whether an SEP event could have been large enough to account for a very enhanced increase in ${ }^{14} \mathrm{C}$ content in cedar trees around $775 \mathrm{AD}$. We examined previous large SEP events and inferred solar activity at that time to rule out the possibility of an SEP origin. The seventh theme was to understand how impulsive Fe-rich SEP events are related to solar flares and CMEs and how we can deduce their coronal origins based on their relative elemental abundances and inferred coronal source temperatures. We compared various parametric properties of a large number of the Fe-rich events with associated flares and CMEs to understand these events better.

\section{INTRODUCTION}

It has been known for more than six decades that the Sun produces transient bursts of solar energetic particle (SEP) events that can have deleterious consequences for human activities. These events consist of protons and ions with energies typically extending to tens of millions of electron volts $(\mathrm{MeV})$, sufficient to produce enhanced electron densities in the ionosphere. Several times per solar cycle the event energies extend to giga-electron volts $(\mathrm{GeV})$, which can be measured by ground-based detectors. The typical SEP events are detected by satellites and envelope the Earth for periods of several hours to several days.

The characteristics of SEP events such as their peak intensities, spectra, and elemental and isotopic compositions have been extensively measured. We now understand that there are two classes of SEP events (Reames, 1999): (1) the impulsive events, defined by low intensities, limited spatial extent, and enhanced heavy element abundances; and (2) the gradual events, defined by high intensities, large spatial extent in interplanetary space, and elemental abundances comparable to those of the solar corona. The first group is associated with small solar flares and the second with coronal shocks driven by fast coronal mass ejections (CMEs). The favored paradigm is that impulsive particle events are produced in short ( $10 \mathrm{~min})$ episodes of local magnetic reconnection in solar active region flares, and the gradual events originate in prolonged ( hours) particle acceleration by broad shock waves driven by fast and wide CMEs. The gradual events that are important for space weather effects are the focus of our interest.

Within this basic picture of SEP events lie many bewildering problems. Perhaps the most challenging problem is to understand which solar energetic events will produce SEP events at Earth and what the characteristics of the resulting events will be. There is a rough correlation of the peak $20 \mathrm{MeV}$ SEP intensities with the speeds of associated CMEs, but for any given CME speed the resulting observed SEP events have a range of 4 to 5 decades of peak intensities. The

Approved for public release; distribution is unlimited. 
problem is to understand what other coronal or interplanetary characteristics play roles in SEP production. We also know that only some solar flares are associated with fast CMEs or SEP events. Are those flares different, and if so, how?

\section{METHODS, ASSUMPTIONS, AND PROCEDURES}

We use observational data taken from several NASA spacecraft experiments to test our understanding of the origin of SEPs. In particular we use the interplanetary 20-MeV proton data from the EPACT instrument on the Wind spacecraft and the $\mathrm{E}>10 \mathrm{MeV}$ data from the NOAA GOES spacecraft. The solar data are taken from ground and space-based observatories, including solar EUV and white-light coronal images and photospheric magnetic field intensities from the NASAESA SOHO satellite and soft X-ray data from the GOES satellite. The general approach is to identify a number of SEP events observed at $1 \mathrm{AU}$ to be used in statistical comparisons with their associated solar properties such as the CMEs, flares, and ambient solar winds.

\section{RESULTS AND DISCUSSION}

\subsection{Effects of Coronal Holes on Properties of SEP Events}

Kunches \& Zwickl (1999) found that the times from flare X-ray maxima to 1 AU onsets of $>25 \mathrm{MeV}$ proton events during 1976-1997 were typically longer by periods of hours when a $\mathrm{CH}$ lay between the CME source region and the $1 \mathrm{AU}$ magnetic footpoint. However, later studies of front-side, west-hemisphere fast halo CMEs by Shen et al. $(2006,2010)$ found that the CH proximities, areas, and locations relative to the CME sources and Earth magnetic connections were not factors in the occurrence of associated SEP events. The two works suggested opposite answers to the basic question of whether CHs play any role in the properties of SEP events, and the question remains controversial.

CH fields may affect coronal/interplanetary SEP production by latitudinally and/or longitudinally deflecting the CME and its preceding shock envelope. This idea is motivated by the recent discovery (Gopalswamy et al., 2009) that some disk-center CMEs produced shocks at $1 \mathrm{AU}$ without accompanying interplanetary CME (ICME) drivers. These CMEs were located adjacent to CHs which deflected the CMEs away from the Sun-Earth line, by angles as large as $\sim 50^{\circ}$ (Gopalswamy et al., 2009, 2010). The magnetic connectivity of the SEP acceleration region to the observer may change from a favorable to an unfavorable one or vice versa, as indicated in the schematic of Figure 1, showing how the pressure of magnetic fields might deflect the propagation direction of a CME. The CME and preceding shock (red lines) that would have propagated toward the observer (blue dot) are deflected eastward (blue lines) by the fields extending from the $\mathrm{CH}$ lying west of the CME. Displacements of SEP shock acceleration regions suggested a new SEP environmental factor worthy of further exploration.

We used timing parameters and peak intensities of $20 \mathrm{MeV}$ SEP from the event list of Table 1 of Kahler (2005). We selected for analysis, besides the peak SEP intensity Ip, the SEP onset time $T_{O}$, defined as the time from CME onset to SEP onset at Wind, and $T_{R}$, the time from SEP onset to half-maximum of the SEP peak intensity. Following Gopalswamy et al. (2010), we calculated for each CME the influence parameter vector $F$, defined as 


$$
F=B^{2} A / r^{2},
$$

where $A$ is the area of the $\mathrm{CH}, B$ is the average magnetic field in the $\mathrm{CH}$, and $r$ is the $\mathrm{CH}$ distance. $F$ for each $\mathrm{CH}$ is directed from the $\mathrm{CH}$ center to the $\mathrm{CME}$ source region, and the total $F$, in units of Gauss squared, is the vector sum of $F$ over all individual CHs. The position angle FPA is the assumed direction in which the $\mathrm{CH}$ fields are deflecting the CME, as indicated for an example SEP event in Figure 2.

We sought a direct connection between SEP parameters and the magnitude or direction of the $F$ vectors, which were resolved into equatorward/poleward and east/west components. We found no statistically significant correlations between these two sets of parameters. An independence of SEP event parameters from the CME propagation directions as defined by their measured PAs (MPAs) was found. The implication here is that even if $\mathrm{CH}$ fields are deflecting CMEs, those deflections are not relevant to the SEP events produced by the CME-driven shocks. An excellent agreement of the CME measured PAs with the flare PAs (fPAs, the angles from Sun center through the flare sites) was found (Figure 3), indicating generally radial propagation of CMEs from the associated flare sites. However, we also found a correlation of PAs of the $F$ vectors (FPAs) with the 41 observed measured PAs consistent with CME deflections in the directions of the $F$ vectors. In summary, the CMEs erupt generally radially, although there is some deflection from radial by the CHs, but the deflections are not important for SEP event intensities and timings. The work (Kahler et al., 2012) supports the idea that CHs are not important for SEP event predictions.

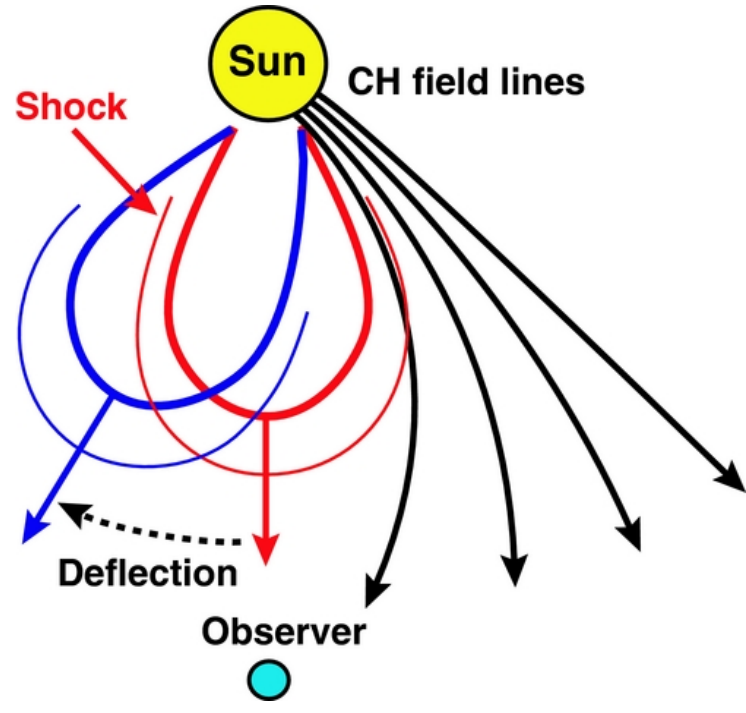

Figure 1: Schematic Diagram of CME Deflection by a Coronal Hole

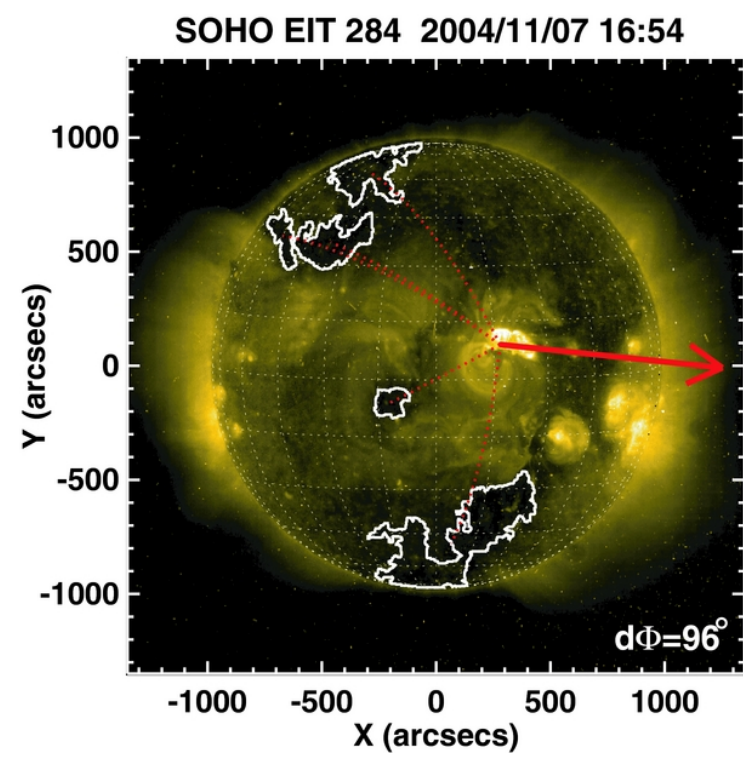

Figure 2: SOHO EIT Image with Coronal Holes and FPA Vector 
We used the same group of $41 \mathrm{E} \approx 20 \mathrm{MeV}$ SEP events with origins near the solar central meridian to search for additional $\mathrm{CH}$ effects. First we investigated whether the presence of a $\mathrm{CH}$ directly between the sources of the CME and of the magnetic connection at $1 \mathrm{AU}$ is an important factor. Then we searched for variations of the SEP events among different solar wind (SW) stream types: slow, fast, and transient. Finally, we considered the separations between CME sources and $\mathrm{CH}$ footpoint connections from 1 AU determined from four-day forecast maps based on Mount Wilson Observatory and the National Solar Observatory synoptic magnetic-field maps and the Wang-Sheeley-Arge model of SW propagation. The observed in-situ magnetic-field polarities and SW speeds at SEP event onsets tested the forecast accuracies employed to select the best SEP/CH connection events for that analysis. Within our limited sample and the three analytical treatments, we found no statistical evidence for an effect of CHs on SEP event peak intensities, onset times, or rise times (Kahler et al., 2014). The only exception was a possible enhancement of SEP peak intensities in magnetic clouds.

\subsection{Size Distributions and Scaling Laws of Solar Flares and SEP Events}

Hudson (1978) pointed out that the size distribution of solar energetic proton (SEP) events is flatter than those of flare electromagnetic emissions. When size distributions are represented by power laws, of the form

$$
d N / d x \sim x^{-\alpha} \text { events/unit } x,
$$

SEP events are found to have values of $\alpha$ that range from $\sim 1.2$ to 1.4 versus values of $\sim 1.8$ to 2.0 for flare electromagnetic emissions. We selected a sample of $\mathrm{E}>10 \mathrm{MeV}$ SEP events with hourly averaged peak fluxes $\geq 1$ proton $\mathrm{cm}^{-2} \mathrm{~s}^{-1} \mathrm{sr}^{-1}$ (or $\geq 1$ proton flux unit (pfu)), above the upper limit peak flux for the smaller "impulsive" SEP events.

Figure 4 contains power-law size distributions for (1) the peak 1-8 $\AA$ fluxes of 540 SXR flares of class $\geq \mathrm{M} 1.0\left(\geq 1.0 \times 10^{-5} \mathrm{~W} \mathrm{~m}^{-2}\right)$ (black line; data from Yashiro et al. (2006); (2) the peak 1-8 $\AA$ fluxes of $52 \geq$ M1.0 SXR flares associated with $>10 \mathrm{MeV}$ proton events (light blue line); (3) the peak 1-8 $\AA$ fluxes of 59 SXR flares associated with CMEs with speeds $\geq 1000 \mathrm{~km} \mathrm{~s}^{-1}$ (red line; Yashiro et al., 2006); and (4) the peak proton fluxes of $58>10 \mathrm{MeV}$ SEP events (reference distribution for slope only, magenta line). The $1000 \mathrm{~km} \mathrm{~s}^{-1} \mathrm{CME}$ speed threshold corresponds roughly to the speeds of CMEs required to produce type II bursts that will extend from the metric to the kilometric wavelength range (Gopalswamy et al., 2005).

We (Cliver et al., 2012) presented evidence that the difference in the slopes of the power-law size distributions of solar flares and SEP events arises primarily because SEP flares, in particular those associated with gradual SEP events, represent an energetic subset of all flares, characterized by fast $\left(\geq 1000 \mathrm{~km} \mathrm{~s}^{-1}\right)$ CMEs. The slope ( $\alpha$ value) of the size distribution for the peak 1-8 A fluxes of SEP- (or fast CME-) associated SXR flares is $\sim 1.3-1.4$, compared to $\sim 1.2$ for the peak proton fluxes of $>10 \mathrm{MeV}$ SEP events and $\sim 2$ for the peak 1-8 $\AA$ fluxes of all SXR flares (Figure 4).

Among many other natural processes, the size distributions of solar X-ray flares and solar energetic particle (SEP) events are scale-invariant power laws. The measured distributions of SEP events proved to be distinctly flatter, i.e., have smaller power-law slopes, than those of the 
flares. This has led to speculation that the two distributions are related through a scaling law, first suggested by Hudson (1978), which implies a direct nonlinear physical connection between the processes producing the flares and those producing the SEP events. We (Kahler, 2013a) presented four arguments against this interpretation. First, a true scaling must relate SEP events to all flare X-ray events, and not to a small subset of the X-ray event population. We also show that the assumed scaling law is not mathematically valid and that although the flare X-ray and SEP event data are correlated, they are highly scattered and not necessarily related through an assumed scaling of the two phenomena. An interpretation of SEP events within the context of a recent model of fractal-diffusive self-organized criticality by Aschwanden (2012) provided a physical basis for why the SEP distributions should be flatter than those of solar flares. These arguments provided evidence against a close physical connection of flares with SEP production.

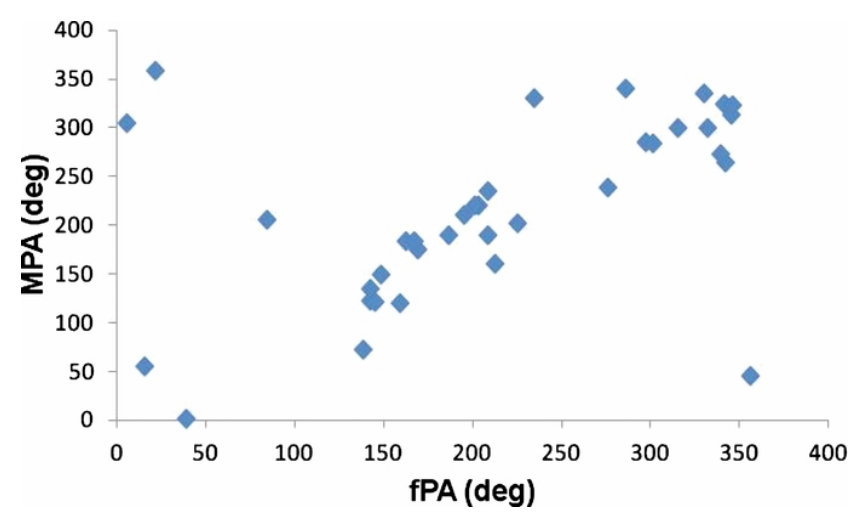

Figure 3: The Coronal Mass Ejection Angles MPA Versus fPA

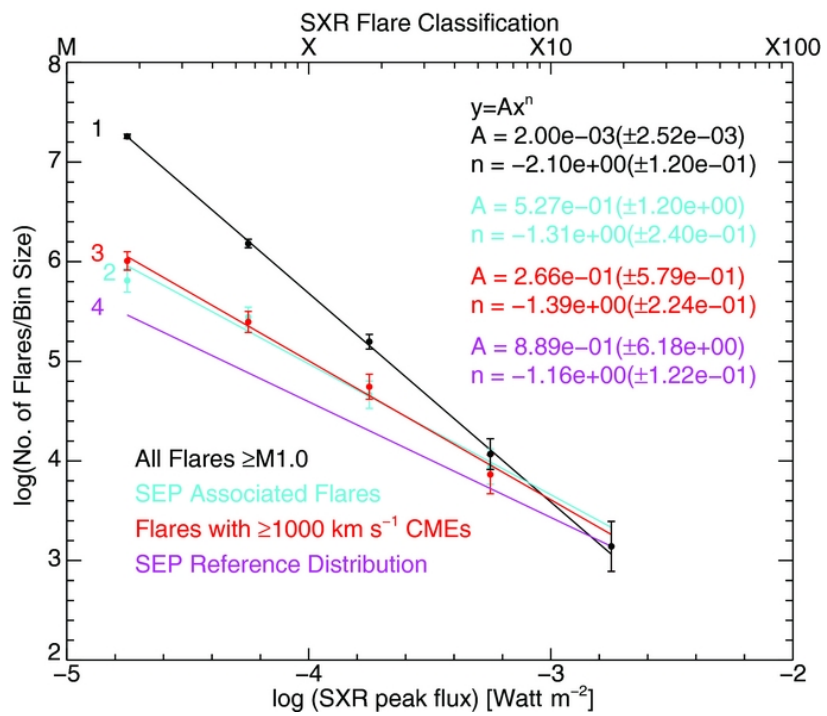

Figure 4: Plot of Size Distributions
of Solar Flares and SEP Events

\subsection{SEP Event Timescales and Associated CME Properties.}

The dependence of SEP event peak intensities Ip on properties of associated CMEs has been extensively examined, but the dependence of SEP event timescales has not been well known. We defined three timescales of $20 \mathrm{MeV}$ SEP events and asked how they are related to speeds $\mathrm{V}_{\mathrm{CME}}$ or widths $\mathrm{W}$ of their associated CMEs observed by LASCO/SOHO. The timescales of the EPACT/Wind $20 \mathrm{MeV}$ events are TO, the onset time from CME launch to SEP onset; TR, the rise time from onset to half the peak intensity ( $0.5 \mathrm{x}$ Ip); and TD, the duration of the SEP intensity above 0.5 Ip. This was a statistical study based on 217 SEP-CME events observed during 1996-2008 (Kahler, 2013b). The large number of SEP events allowed us to examine the 
SEP-CME relationship in five solar-source longitude ranges. In general, we statistically found that $\mathrm{TO}$ declines slightly with $\mathrm{V}_{\mathrm{CME}}$, and TR and TD increase with both $\mathrm{V}_{\mathrm{CME}}$ and $\mathrm{W}$. TO was inversely correlated with log Ip, as expected from a particle background effect. We discussed the implications of this result and found that a background-independent parameter TO+TR also increases with $V_{\text {CME }}$ and $W$ (Figure 5). The correlations generally fell below the $98 \%$ significance level, but there was a significant correlation between $\mathrm{V}_{\mathrm{CME}}$ and $\mathrm{W}$ which renders interpretation of the timescale results uncertain. We suggest that faster (and wider) CMEs drive shocks and accelerate SEPs over longer times to produce the longer TR and TD SEP timescales. The SEP event durations TD were averaged over longitudinal ranges and compared with CME speeds and widths (Figure 6).
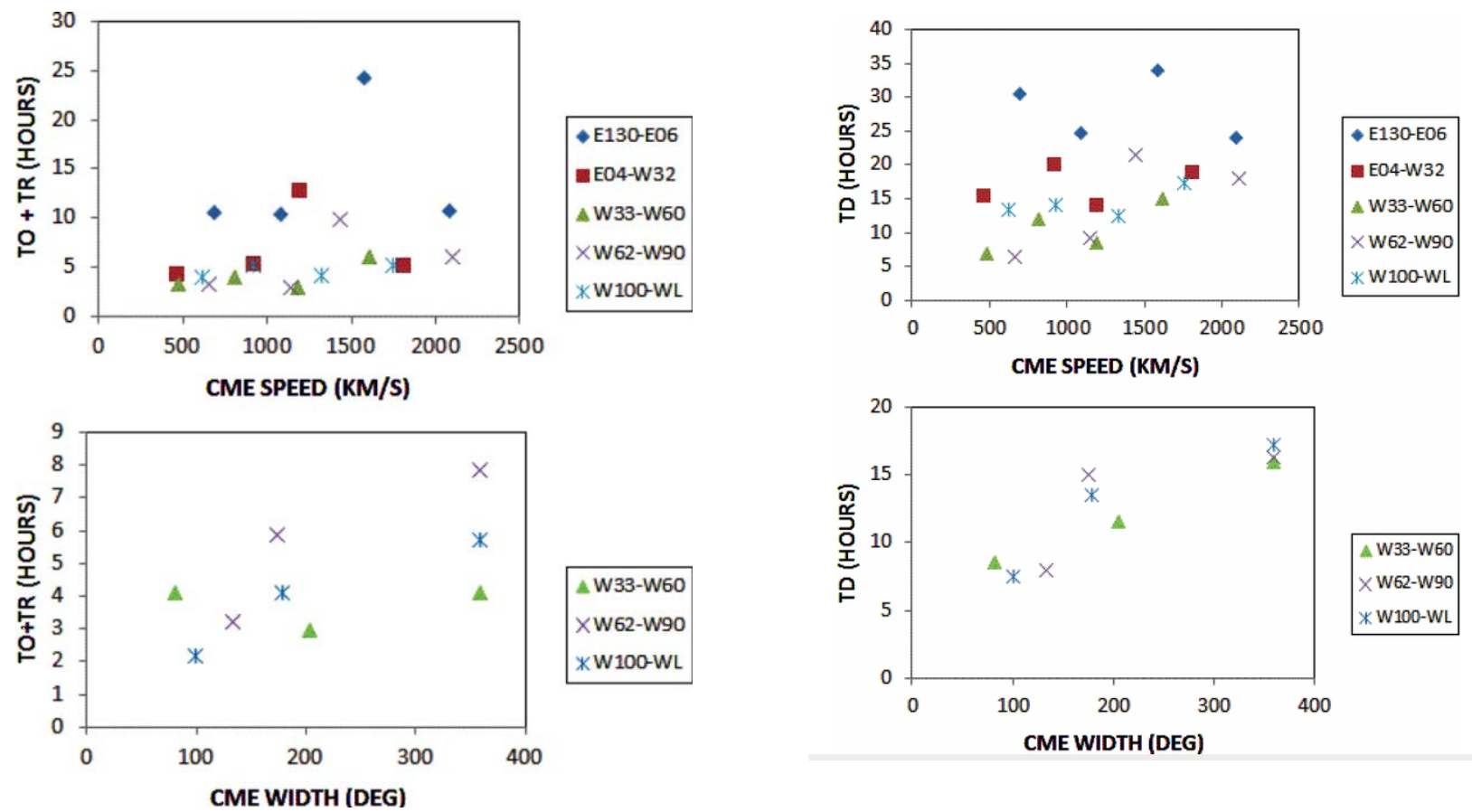

Figure 5: Plot of SEP Onset and Rise Time Medians versus CME Speeds and Widths

\section{Figure 6: Plot of SEP Duration Time Medians versus CME Speeds and Widths}




\subsection{Comparison of the Intensities and Energies of Gradual SEP Events with the Dynamical Properties of Associated CMEs}

Gradual SEP events observed at 1 AU are produced by shocks driven by CMEs. Characterizations of the remotely imaged CMEs and of their associated SEP events observed in situ can be used to increase our ability to forecast SEP events and to understand better the physical connections between the two phenomena. We carried out a statistical comparison of the peak intensities Ip20, of 120 western-hemisphere $20 \mathrm{MeV}$ SEP events with those of their associated CMEs observed by the Solar and Heliospheric Observatory/Large Angle and Spectrometric Coronagraph over the past solar cycle. For a subset of 96 events observed with the EPACT instrument on the Wind spacecraft we also compared the SEP $2 \mathrm{MeV}$ peak intensities Ip2, power-law energy spectral exponents $\gamma$, total SEP energies Esep, and $2 \mathrm{MeV} n \mathrm{cc}^{-1} \mathrm{H} / \mathrm{He}$ ratios with CME properties (Kahler and Vourlidas, 2013). New analyses of white-light CME images enabled us to improve calculations of the CME masses and potential energies and then to determine two values of their kinetic energies based on frontal $V(\mathrm{fr})$ and center-of-mass $V(\mathrm{~cm})$ speeds. Despite considerable scatter in the SEP and CME data, the large dynamical ranges of both the SEP and CME parameters allowed us to determine statistical trends in the comparisons of the logs of the parameters. Ip2, Ip20, and Esep were significantly correlated with CME kinetic energies, masses, and speeds (Figure 7), while $\gamma$ trended lower (harder). Those correlations were higher with $V(\mathrm{fr})$ than with $V(\mathrm{~cm})$ parameters, indicating a less significant role for the body of the CME than for the CME front in SEP production. The high ratios $(\geq 10 \%)$ of Esep to CME energies found by Mewaldt et al.(2008) were confirmed, and the fits are consistent with a linear relationship between the logs of the two energies (Figure 8). The $2 \mathrm{MeV}$ nuc ${ }^{-1} \mathrm{H} / \mathrm{He}$ ratios decrease with increasing CME speeds, which may be an effect of shock geometry. 

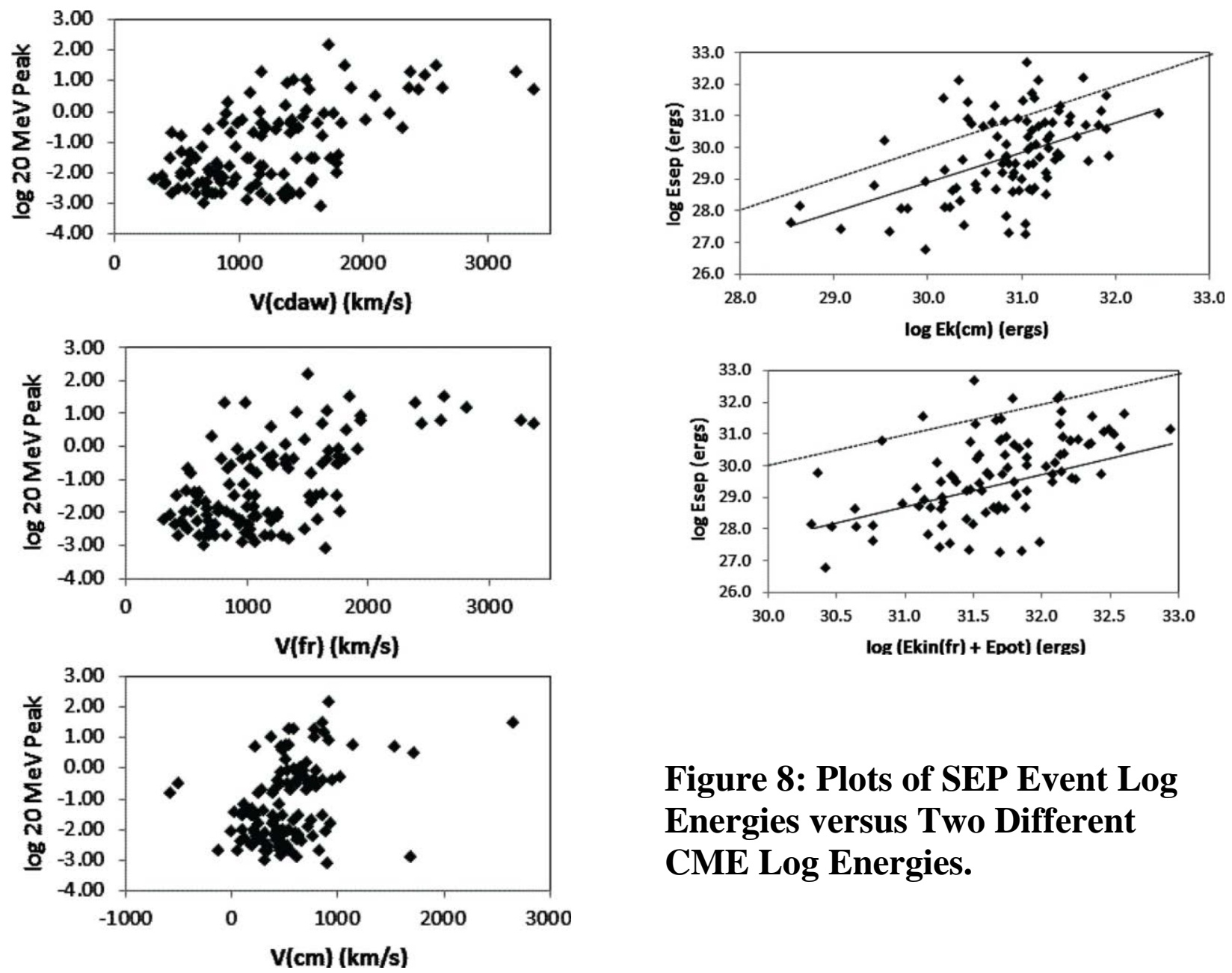

Figure 8: Plots of SEP Event Log Energies versus Two Different CME Log Energies.

Figure 7: Plots of the Peak $20 \mathrm{MeV}$ Proton Intensities versus Three Sets of CME Speeds.

\subsection{The Roles of Interacting CMEs and Solar Wind Streams in SEP Events}

Gradual SEP events are produced in shocks driven by fast and wide CMEs. With a set of 96 western hemisphere $20 \mathrm{MeV}$ SEP events, we tested the possibility that SEP peak intensities, Ip, are enhanced by interactions of their associated CMEs with preceding CMEs (preCMEs) launched during the previous $12 \mathrm{hr}$ (Li et al., 2012). Among SEP events with no, 1, or 2 or more $(2+)$ preCMEs, we found enhanced Ip for the groups with preCMEs, but no differences in TO+TR, the time from CME launch to SEP onset and the time from onset to SEP half-peak Ip. Neither the timings of the preCMEs relative to their associated CMEs nor the preCME widths 
$W_{\text {pree }}$, speeds $V_{\text {pre, }}$ or numbers correlated with the SEP Ip values. The $20 \mathrm{MeV}$ Ip of all the preCME groups correlated with the $2 \mathrm{MeV}$ proton background intensities (Figure 9), consistent with a general correlation with possible seed particle populations. Furthermore, the fraction of CMEs with preCMEs also increased with the $2 \mathrm{MeV}$ proton background intensities. This implies that the higher SEP Ip values with preCMEs may not be due primarily to CME interactions, such as the "twin-CME" scenario, but are explained by a general increase of both background seed particles and more frequent CMEs during times of higher solar activity. This explanation is not supported by our analysis of $2 \mathrm{MeV}$ proton backgrounds in two earlier preCME studies of SEP events, so the relevance of CME interactions for larger SEP event intensities remains unclear (Kahler and Vourlidas, 2014a).

We examined statistically some properties of $9620 \mathrm{MeV}$ gradual SEP events as a function of three different types of solar wind (SW). Gradual SEP (E $>10 \mathrm{MeV}$ ) events are produced in shocks driven by fast $\left(\mathrm{V}>900 \mathrm{~km} \mathrm{~s}^{-1}\right)$ and wide $\left(\mathrm{W}>60^{\circ}\right) \mathrm{CMEs}$. We found no differences among the transient, fast, and slow SW streams for SEP $20 \mathrm{MeV}$ proton event timescales. It has recently been found that the peak intensities Ip of these SEP events scale with the $2 \mathrm{MeV}$ proton background intensities (Kahler and Vourlidas, 2014a), which may be a proxy for the nearSun shock seed particles. Both the intensities Ip and their $2 \mathrm{MeV}$ backgrounds were significantly enhanced in transient SW compared to those of fast and slow SW streams (Figure 10), and the values of Ip normalized to the $2 \mathrm{MeV}$ backgrounds only weakly correlated with CME V for all SW types. This result implies that forecasts of SEP events could be improved by monitoring both the Sun and the local SW stream properties. We interpreted an observed correlation between CME V and the $2 \mathrm{MeV}$ background for SEP events in transient SW as a manifestation of enhanced solar activity (Kahler and Vourlidas, 2014b).

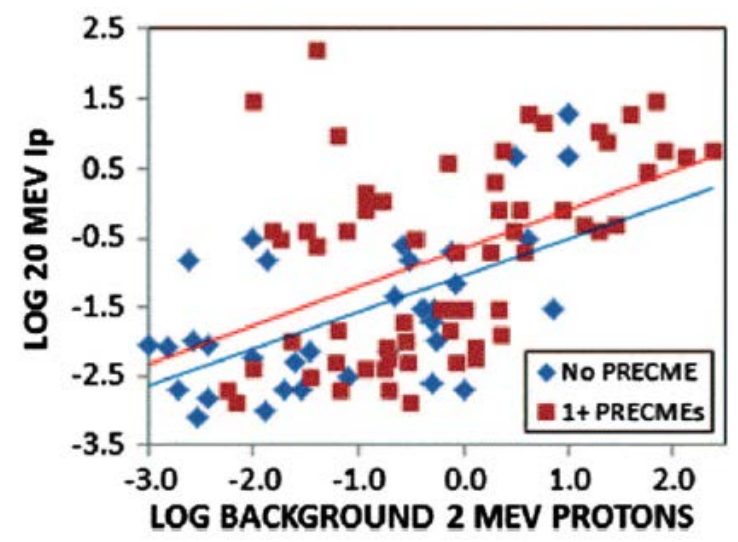

Figure 9. Plots of $\log$ Ip versus $\log 2$ $\mathrm{MeV}$ proton backgrounds.

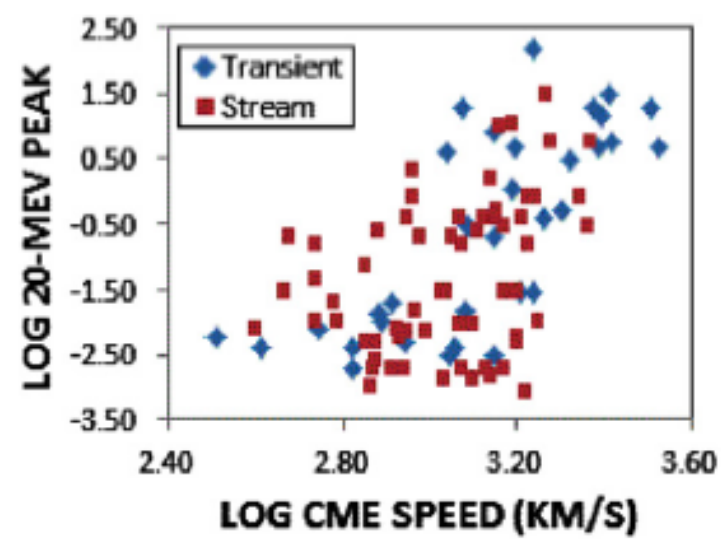

Figure 10. Plots of $\log$ Ip versus log CME speeds. 


\subsection{The Cosmogenic Nuclide Event of 775 AD}

From high-time-resolution (1-2 yr) measurements of ${ }^{14} \mathrm{C}$ in Japanese cedar trees from $750-820$ AD, Miyake et al. (2012) reported a 12\% increase of carbon content from 774-775 AD. Miyake et al. argued that both a nearby supernova and the Sun were improbable sources for the $775 \mathrm{AD}$ event. However, a 45 times multiple of the hard proton spectrum of the 23 February 1956 SEP event has been proposed for the 775 event. Given the current interest in determining the limits of extreme solar activity and the range of proposed origins for the 775 event, we examined the spectrum of the 1956 ground level event (GLE; requiring $>500 \mathrm{MeV}$ protons) used to model the $775 \mathrm{AD}$ cosmogenic nuclide event and reviewed the solar circumstances that gave rise to the 1956 SEP event. We considered the production of large $>30 \mathrm{MeV}\left(F_{30}\right)$ events by the Sun during the modern era and the flare and active region sizes required to produce the 775 event. We explored requirements for a SEP event capable of producing the cosmogenic nuclide event of 775 A.D., and reviewed solar circumstances at that time.

A solar source for the $775 \mathrm{AD}$ event would require a $>1 \mathrm{GV}$ spectrum $\sim 45$ times stronger than that of the intense high-energy SPE of 1956 February 23. This implies a $>30 \mathrm{MeV}$ proton fluence $\left(F_{30}\right)$ of $\sim 8 \times 10^{10}$ proton $\mathrm{cm}^{-2}, \sim 10$ times larger than that of the strongest 3 month interval of SPE activity in the modern era. This inferred $F{ }_{30}$ value for the 775 SPE is inconsistent with the occurrence probability distribution for $>30 \mathrm{MeV}$ SEP events. The 775 event occurred during a period of relatively low solar activity, with a peak smoothed amplitude about half that of the second half of the 20th century. The $\sim 1945-1995$ interval, the most active of the last $\sim 2000 \mathrm{yr}$, failed to witness an SEP event comparable to that required for the proposed solar event in 775 . These considerations (Cliver et al., 2014) challenged the recent suggestion that the 775 event is likely of solar origin.

\subsection{Abundance Enhancements in Impulsive SEP Events}

We studied the abundances of the elements He through $\mathrm{Pb}$ in Fe-rich impulsive solar energeticparticle (SEP) events with measurable abundances of ions with atomic number, $\mathrm{Z}>2$, and their relationship with coronal mass ejections (CMEs) (Reames et al., 2014a). On average the element abundances in these events are similar to coronal abundances at low $\mathrm{Z}$ but, for heavier elements, enhancements rise as a power law in the mass-to-charge ratio $A / Q$ of the ions (at coronal temperatures of 2.5-3 MK) to a factor of 3 at Ne, 9 at Fe, and 900 for $76 \leq Z \leq 82$. The 111 of these Fe-rich impulsive SEP events we found, between November 1994 and August 2013 using the Wind spacecraft, have a 74\% association rate with CMEs. The CMEs have a median width of $71^{\circ}$, and are characteristically from the western hemisphere on the Sun with a median speed of $650 \mathrm{~km} \mathrm{~s}^{-1}$. The faster $\left(>700 \mathrm{~km} \mathrm{~s}^{-1}\right)$, wider CMEs in our sample are related to SEPs with coronal abundances indicating hot coronal plasma with fully ionized $\mathrm{He}, \mathrm{C}, \mathrm{N}$, and $\mathrm{O}$ and moderate enhancements of heavier elements relative to He, but slower $\left(<700 \mathrm{~km} \mathrm{~s}^{-1}\right)$, narrower CMEs emerge from cooler plasma where higher SEP mass-to-charge ratios, $A / Q$, yield much greater abundance enhancements.

We extended the study to event-to-event variations in the abundance enhancements of the elements He through $\mathrm{Pb}$ for Fe-rich impulsive solar energetic-particle (SEP) events, and their relationship with properties of associated coronal mass ejections (CMEs) and solar flares 
(Reames et al., 2014b). Using a least-squares procedure we fit the power-law enhancement of element abundances as a function of their mass-to-charge ratio $A / Q$ to determine both the power index and the coronal temperature (which determines $Q$ ) in each of 111 impulsive SEP events identified previously. Individual SEP events with the steepest element enhancements, e.g. $\sim(A / Q)^{6}$, tend to be smaller, lower-fluence events with steeper energy spectra that are associated with B- and C-class X-ray flares (see Figure 11), with cooler ( 2.5 MK) coronal plasma, and with narrow $\left(<100^{\circ}\right)$, slower $\left(<700 \mathrm{~km} \mathrm{~s}^{-1}\right)$ CMEs. On the other hand, higher-fluence SEP events have flatter energy spectra, less-dramatic heavy-element enhancements, e.g. $\sim(A / Q)^{3}$, and come from somewhat hotter coronal plasma ( 3.2 MK) associated with C-, M- and even X-class $\mathrm{X}$-ray flares and with wider CMEs. These results suggest a continuum of events rather than the generally accepted two-class structure (Reames, 1999).

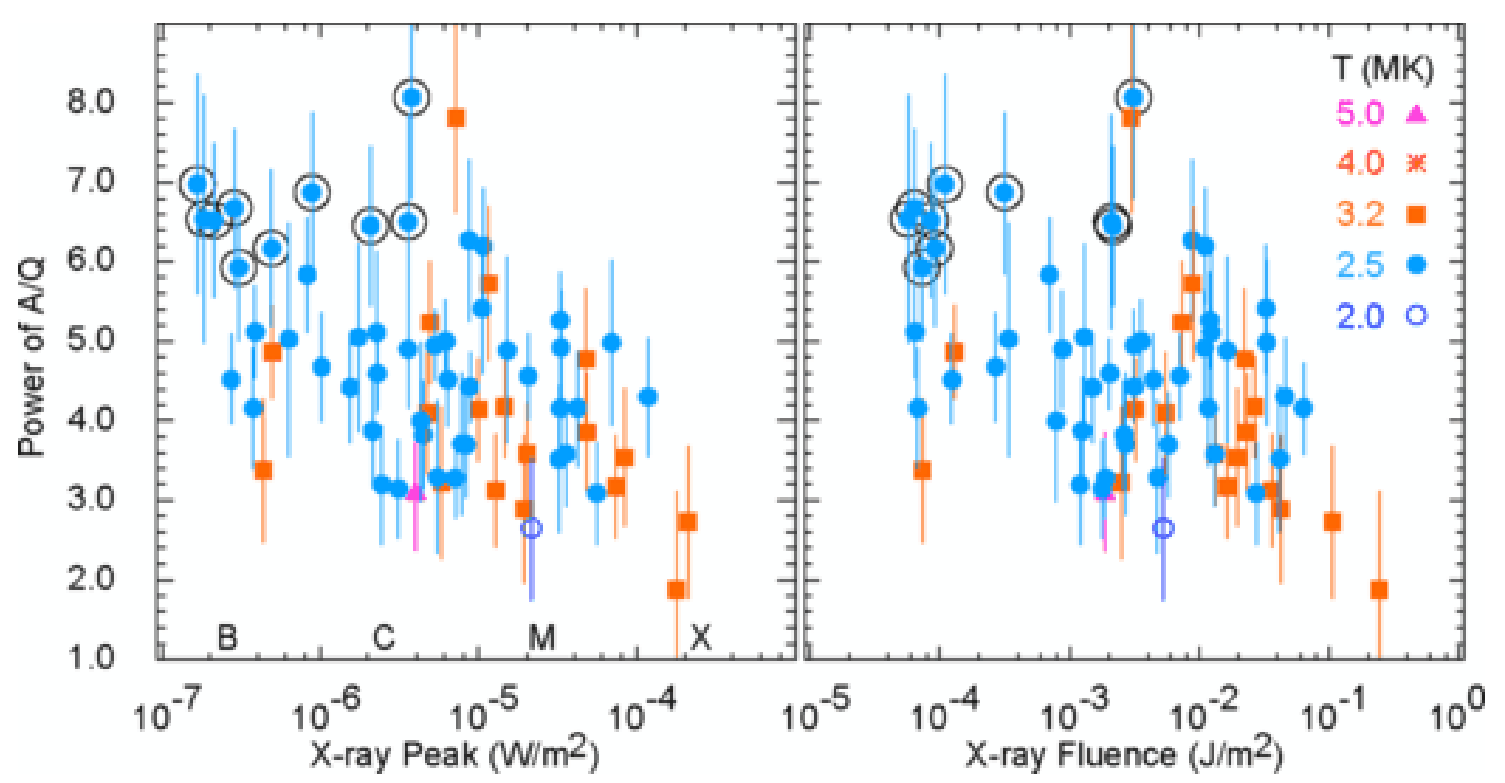

Figure 11. Plots of the Power of $A / Q$ versus $X$-ray Flare Peak and Fluence. 


\section{CONCLUSIONS}

The work described in the previous section centers on the basic paradigm of SEP production by CME-driven shocks. The goal is to expand our understanding of the SEP events by focusing on well defined aspects of those events. We ask whether or how coronal holes, adjacent to fast CMEs driving the shocks, can modify the resulting SEP events in any obvious way. SEP events are organized as power laws in peak intensities, and this was the basis for two works to find some insights into that organization. The relationship of CMEs to SEP events is fundamental to understanding how CME energy is transformed into SEP energy, so we compared energies of SEP events with those of CMEs as measured with different physical assumptions. The environment in which SEPs are generated and propagate to $1 \mathrm{AU}$ was also the basis of several studies. One dealt with a popular model for CME-CME interactions and the other looked at the kind of solar wind in which SEPs propagate. The coronal source material and the heavy element enhancements of Fe-rich SEP events was determined from a study starting with impulsive SEP events not produced in CME shocks. An overview of the work is given in the Summary section and is then followed by a concise description of the research tasks in the Results and Discussion section along with references to the published work. 


\section{REFERENCES}

Aschwanden, M. J., “A statistical fractal-diffusive avalanche model of a slowly-driven selforganized criticality system,” Astron. Astrophys., 539, 2012, id.A2, pp. 1-15.

Cliver, E. W., Tylka, A. J., Dietrich, W. F., and Ling, A. G., "On a Solar Origin for the Cosmogenic Nuclide Event of 775 A.D.,” Astrophys. J., 781, 2014, 32, pp. 1-4.

Cliver, E. W., Ling, A. G., Belov, A., and Yashiro, S., "Size Distributions of Solar Flares and Solar Energetic Particle Events,” Astrophys. J. Let., 756, 2012, L29-L31.

Gopalswamy, N., Aguilar-Rodriguez, E., and Yashiro, S., et al., “Type II radio bursts and energetic solar eruptions,” J. Geophys. Res., 110, 2005, A12S07.

Gopalswamy, N., Makela, P., Xie, H., Akiyama, S., and Yashiro, S., “CME interactions withcoronal holes and their interplanetary consequences,” J. Geophys. Res., 114, 2009, A00A22.

Gopalswamy, N., Makela, P., Xie, H., Akiyama, S., and Yashiro, S., "Solar Sources of 'Driverless’ Interplanetary Shocks,” Twelfth Int. Solar Wind Conf. (AIP Conf. Proc. 1216), ed. M. Maksimovic, et al., (Melville, NY: AIP), 2010, pp. 452-458.

Hudson, H. S., “Threshold effect in second-stage acceleration,” Sol. Phys., 57, 1978, pp. 237240.

Kahler, S. W., Akiyama, S., and Gopalswamy, N., "Deflections of Fast Coronal Mass Ejections and the Properties of Associated Solar Energetic Particle Events,” Astrophys. J., 754, 2012, 100, pp. 1-7.

Kahler, S. W., Arge, C. N., Akiyama, S., and Gopalswamy, N., "Do Solar Coronal Holes Affect the Properties of Solar Energetic Particle Events?” Solar Phys., 289, 2014, pp. 657-673.

Kahler, S. W., "Characteristic Times of Gradual Solar Energetic Particle Events and Their Dependence on Associated Coronal Mass Ejection Properties,” Astrophys. J., 628, 2005, pp. 1014-1022.

Kahler, S. W., "Does a Scaling Law Exist between Solar Energetic Particle Events and Solar Flares?” Astrophys. J., 769:35, 2013a, pp. 1-5.

Kahler, S. W., “A Comparison of Solar Energetic Particle Event Timescales with Properties of Associated Coronal Mass Ejections,” Astrophys. J., 769, 2013b, 110, pp. 1-10.

Kahler, S. W. and Vourlidas, A., "A Comparison of the Intensities and Energies of Gradual Solar Energetic Particle Events with the Dynamical Properties of Associated Coronal Mass Ejections," Astrophys. J., 769, 2013, 143, pp. 1-12.

Kahler, S. W. and Vourlidas, A., "Do Interacting Coronal Mass Ejections Plan a Role In Solar Energetic Particle Events?” Astrophys. J., 784, 2014a, 47, pp. 1-10. 
Kahler, S. W. and Vourlidas, A., "Solar Energetic Particle Events in Different Types of Solar Wind,” Astrophys. J., 2014b, 791, 4, pp. 1-8.

Kunches, J. M. and Zwickl, R. D., "The effects of coronal holes on the propagation of solar energetic protons,” Rad. Meas., 30, 1999, pp. 281-286.

Li, G., Moore, R., Mewaldt, R. A., Zhao, L., and Labrador, A. W., “A Twin-CME Scenario for Ground Level Enhancement Events,” Space Sci. Rev., 171, 2012, 1-4, pp. 141-160.

Mewaldt, R. A., Cohen, C. M. S., Giacalone, J., Mason, G. M., Chollet, E. E., Desai, M. I., Haggerty, D. K., Looper, M. D., Selesnick, R. S., and Vourlidas, A., “How Efficient are Coronal Mass Ejections at Accelerating Solar Energetic Particles?” Particle Acceleration and Transport in the Heliosphere and Beyond: 7th Annual International Astrophysics Conference, AIP Conference Proceedings, 2008, Volume 1039, pp. 111-117.

Reames, D. V., "Particle acceleration at the Sun and in the heliosphere,” Space Sci. Rev., 90, 1999, pp. 413-491.

Reames, D. V., Cliver, E. W., and Kahler, S. W., "Abundance Enhancements in Impulsive Solar Energetic-Particle Events with Associated Coronal Mass Ejections,” Solar Phys., 289, 2014a, pp. 3817-3841.

Reames, D. V., Cliver, E. W., and Kahler, S. W., "Variations in Abundance Enhancements in Impulsive Solar Energetic-Particle Events and Related CMEs and Flares,” Solar Phys., 2014b, in press.

Shen, C., Wang, Y., Ye, P., and Wang, S., "Is There Any Evident Effect of Coronal Holes on Gradual Solar Energetic Particle Events?” Astrophys. J., 639, 2006, pp. 510-515.

Shen, C., Yao, J., and Wang, Y.-M., et al., "Influence of coronal holes on CMEs in causing SEP events,” Res. Astron. Astrophys., 10, 2010, pp. 1049-1060.

Yashiro, S., Akiyama, S., Gopalswamy, N., and Howard, R. A., "Different Power-Law Indices in +the Frequency Distributions of Flares with and without Coronal Mass Ejections,” Astrophys. J., 650, 2006, L143-L146. 


\section{DISTRIBUTION LIST}

\section{DTIC/OCP}

8725 John J. Kingman Rd, Suite 0944

Ft Belvoir, VA 22060-6218

$1 \mathrm{cy}$

AFRL/RVIL

Kirtland AFB, NM 87117-5776

2 cys

Official Record Copy

AFRL/RVBXS/Dr. Stephen Kahler

$1 \mathrm{cy}$ 